\title{
Complicações na facoemulsificação interferem no aparecimento da degeneração macular relacionada à idade?
}

\author{
Does phacoemulsification complications interfere in the \\ appearance of age related macular degeneration?
}

Angela Bettarello', Wagner Ghirelli', Tadeu Cvintal ${ }^{3}$

\section{Resumo}

Objetivo: Avaliar se uma cirurgia de catarata por facoemulsificação com complicação intra-operatória é um fator de risco para o desenvolvimento de degeneração macular relacionada à idade. Métodos: Estudo retrospectivo longitudinal tipo caso-controle com revisão dos prontuários de 1150 pacientes submetidos à facectomia no período de 1995 a 1999, realizadas por apenas um cirurgião (T.C.). Para cálculo estatístico foram utilizados o Qui-quadrado com correção de Yates, Teste Exato de Fisher e Teste de MannWhitney, com intervalo de confiança de $95 \%(\mathrm{p}<0,05)$. Resultados: Em 224 pacientes foram encontrados 297 olhos operados, com seguimento médio de 6,15 anos. Das cirurgias realizadas, 240 foram consideradas normais e 57 complicadas. Das cirurgias complicadas no sexo feminino, num total de 38 cirurgias, 10 apresentaram alterações em F.O. $(p=0,0509)$. Das cirurgias realizadas em pacientes do sexo masculino 19 foram complicadas, e destas, apenas um olho evoluiu com F.O. alterado $(p=0,5737)$. Conclusão: Não foi observado aumento, estatisticamente significante, da incidência de degeneração macular em olhos que tiveram complicação intra-operatória. Mais estudos são necessários para melhor elucidação da patogenia desta patologia.

Descritores: Degeneração macular; Extração de catarata; Facoemulsificação/métodos; Neovasularização coroidal; Complicações intra-operatórias; Fatores etários

\footnotetext{
${ }^{1}$ Fellow do Setor de Retina e Vítreo do Instituto de Oftalmologia Tadeu Cvintal - São Paulo (SP) - Brasil;

${ }^{2}$ Chefe do departamento de Retina e Vítreo do Instituto de Oftalmologia Tadeu Cvintal - São Paulo (SP) - Brasil;

${ }^{3}$ Diretor do Instituto de Oftalmologia Tadeu Cvintal - São Paulo (SP) - Brasil.

Trabalho realizado no Instituto de Oftalmologia Tadeu Cvintal - São Paulo (SP) - Brasil

Recebido para publicação em: 27/05/07 - Aceito para publicação em 28/08/07
} 


\section{INTRODUÇÃO}

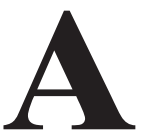
degeneração macular relacionada à idade (DMRI) é a principal causa de deficiência visual severa e irreversível em países desenvolvidos e sua incidência e prevalência aumentam com a idade $^{(1)}$.

A DMRI é uma desordem da mácula caracterizada por um ou mais dos seguintes achados: 1 - formação de drusas; 2- anormalidades do epitélio pigmentar da retina (EPR) como hiper ou hipopigmentação; 3- atrofia geográfica do EPR envolvendo o centro da fóvea; e 4membrana neovascular coroidal ${ }^{(1)}$.

Embora continue com sua patogenia pouco compreendida $^{(2)}$, muitos fatores de risco, como tabagismo, aterosclerose, hipertensão arterial, diabetes mellitus, inflamação, exposição a ultravioleta, íris clara e cirurgia de catarata têm sido descritos, porém, exceto a idade e o fator genético, que são mais consistentes ${ }^{(3)}$, outros ainda precisam de comprovação, e permanecem em investigação ${ }^{(1)}$.

A relação entre a cirurgia de catarata e a DMRI tem sido inconsistente ${ }^{(4)}$. Enquanto alguns estudos epidemiológicos têm sugerido que a cirurgia aumente a incidência e a progressão, outros não têm observado associação ${ }^{(4)}$.

Tentamos com este trabalho responder a seguinte pergunta: uma cirurgia de catarata por facoemulsificação com complicação intra-operatória seria um fator de risco para DMRI?

\section{Métodos}

Estudo retrospectivo longitudinal tipo caso-controle com revisão dos prontuários de 1150 pacientes submetidos à facectomia, no período de 1995 a 1999, realizadas por apenas um cirurgião (T.C.).

Os critérios de inclusão deste trabalho foram: 1idade maior que 40 anos de idade na data da cirurgia; 2 facectomia por facoemulsificação realizada por um cirurgião; 3- fundo de olho normal no pré-operatório; 4tempo de seguimento maior que três anos.

Os critérios de exclusão deste trabalho foram: 1idade menor de 40 anos na data da cirurgia; 2 - facectomia por extração extra ou intracapsular; 3-presença de patologia retiniana como retinopatia diabética, oclusão vascular, uveíte, maculopatia de qualquer etiologia ou alta miopia (acima de seis dioptrias); 4- patologias corneanas como ceratocone, transplante de córnea, cirurgias tríplices; e 5 pacientes com seguimento menor que três anos.
Foram selecionados 224 prontuários, sendo avaliado: sexo, idade, fundo de olho (F.O.) pré-operatório normal para inclusão, relato de complicações intra-operatórias, F.O. pós-operatório e tempo de seguimento.

O F.O. pós-operatório foi considerado alterado quando do surgimento de um ou mais sinais da DMRI ${ }^{(1)}$ observados no exame clínico do paciente, por um dos dois médicos (T.C. ou W.G.)

Foram consideradas complicações intra-operatórias os seguintes achados: a- colocação de lente intraocular no recesso; b- diálise de zônula; c- ruptura de cápsula com ou sem perda vítrea e d- núcleo no vítreo.

As lentes intra-oculares utilizadas foram de silicone e de polimetilmetacrilato (PMMA), sem proteção para ondas de comprimento pequeno (luz azul).

Para cálculo estatístico foram utilizados o Quiquadrado com correção de Yates, Teste Exato de Fisher e Teste de Mann-Whitney, com intervalo de confiança de $95 \%(\mathrm{p}<0,05)$.

\section{Resultados}

Dos 224 pacientes 73 eram do sexo masculino e 151 do sexo feminino. A idade variou de 40 a 91 anos, tendo uma média geral de 70,02 anos. Dos 224 pacientes foram encontrados 297 olhos operados, com seguimento médio de 6,15 anos. Destes 297 olhos, 202 eram de pacientes do sexo feminino, e 95 de pacientes do sexo masculino.

Das cirurgias realizadas, 240 foram consideradas normais e 57 complicadas. F.O. alterado foi encontrado em 37 olhos, sendo 11 em pacientes com cirurgia complicada (Tabela 1). Com $\mathrm{p}=0,1294$.

Das cirurgias realizadas em pacientes do sexo feminino (tabela 2), 164 foram sem complicações, destas 20 apresentaram alterações em F.O. Das cirurgias complicadas, num total de 38 cirurgias, 10 apresentaram alterações em F.O., com $\mathrm{p}=0,0509$.

Das cirurgias realizadas em pacientes do sexo masculino, 19 foram complicadas, e destas, apenas um olho evoluiu com F.O. alterado ( $\mathrm{p}=0,5737)$. Das $76 \mathrm{ci-}$ rurgias normais, 6 pacientes desenvolveram alteração (tabela 3).A média de idade entre o grupo que teve cirurgia complicada e apresentou F.O. normal foi de 68,17 anos, e a média de idade do grupo que também teve cirurgia complicada e apresentou F.O. alterado foi de 72,72 anos, com $\mathrm{p}=0,1171$, não sendo, estatisticamente significante.

Assim como a idade, o tempo de seguimento foi semelhante entre os grupos, tendo o grupo que teve ci- 
rurgia complicada e desenvolvimento de DMRI um seguimento médio de 5, 90 anos, enquanto o grupo que teve cirurgia complicada e não desenvolveu alteração teve um seguimento médio de 5,97 anos.

\section{Dıscussão}

A coexistência de catarata e DMRI é freqüente, principalmente em pacientes de maior idade ${ }^{(5)}$. A maior causa de baixa de visão em idosos é a catarata, enquanto que a maior causa de cegueira irreversível é a DMRI ${ }^{(6)}$.

Existem duas teorias para explicar o aparecimento de DMRI. A primeira por alterações metabólicas oxidativas, que ocorrem no $\operatorname{EPR}^{(7)}$ e a segunda por alterações vasculares na coriocapilar ${ }^{(8)}$.

Acredita-se ainda que as prostaglandinas possam estar envolvidas na auto-regulação da circulação retiniana. Existe um feedback positivo entre radicais livres e ciclooxigenase ${ }^{(9)}$.

Apesar de muitos estudos terem sugerido uma possível associação da cirurgia de catarata e o subseqüente desenvolvimento de DMRI neovascular, nenhum estudo avaliou essa relação levando em conta os achados maculares pré-operatórios, como presença de drusas grandes e moles ou alterações pigmentares da retina ${ }^{(10)}$.

Este estudo retrospectivo realizado com pacientes submetidos à facoemulsificação demonstrou que não há uma relação entre cirurgia de catarata complicada e aumento da ocorrência de DMRI.

Entretanto, devido ser um estudo retrospectivo, dados como coloração da íris e antecedentes familiares não constavam em grande parte dos prontuários vistos, e não puderam ser analisados neste estudo. Porém, com o grande número da amostra, esses fatores genéticos podem ser considerados equivalentes entre os grupos avaliados. Embora a raça não tenha sido analisada, esse estudo foi realizado com pacientes de clínica privada, onde há predomínio da raça branca.

Após excluir fatores que poderiam nos induzir ao erro, como por exemplo, cirurgiões diferentes, tempo de seguimento diferente e, principalmente, idades diferentes, observamos que não houve alteração fundoscópica, estatisticamente significante, em nenhum dos grupos, quer tivessem cirurgia complicada, quer cirurgia normal.

Deve-se salientar, entretanto, que quando separados quanto ao sexo, os grupos mostraram resultados diferentes, podendo ser justificado pelo grande número de pacientes do sexo feminino, e principalmente pela pequena amostra de pacientes do sexo masculino que tiveram cirurgia complicada e desenvolveram DMRI.
Tabela 1

Relação de F.O. alterado x cirurgia complicada

\begin{tabular}{|c|c|c|c|}
\hline Cirurgia $\quad$ F.O. & Normal & Alterado & Total \\
\hline Normal & 214 & 26 & 240 \\
\hline Complicada & 46 & 11 & 57 \\
\hline Total & 260 & 37 & 297 \\
\hline
\end{tabular}

Tabela 2

Relação de F.O. alterado x cirurgia complicada quanto ao sexo feminino

\begin{tabular}{l|rrr}
\hline Cirurgia & Formal & Alterado & Total \\
\hline Normal & & & \\
Complicada & 144 & 20 & 164 \\
Total & 28 & 10 & 38 \\
\hline
\end{tabular}

Tabela 3

Relação de F.O. alterado x cirurgia complicada quanto ao sexo masculino

\begin{tabular}{|c|c|c|c|}
\hline Cirurgia $\quad$ F.O. & Normal & Alterado & Total \\
\hline Normal & 70 & 06 & 76 \\
\hline Complicada & 18 & 01 & 19 \\
\hline Total & 88 & 07 & 95 \\
\hline
\end{tabular}

Alguns autores observaram que a relação entre a cirurgia de catarata, a progressão da DMRI, e a incidência de DMRI avançada, permaneceu significante mesmo após ajuste para tabagismo, ingestão de bebida alcoólica, consumo de vitamina, hipertensão arterial e pressão de perfusão ${ }^{(2)}$.

Em um estudo retrospectivo com 25 olhos com DMRI submetidos à facoemulsificação, observaram melhora da acuidade visual aaaem $72 \%$ dos olhos, e nenhuma deterioração da visão dos pacientes, nem evolução do quadro degenerativo ${ }^{(11)}$.

A progressão da degeneração macular foi observada em pacientes submetidos à cirurgia de catarata ${ }^{(6)}$, porém a razão que explica essa associação ainda não está estabelecida.Alguns fatores são possíveis, entre eles o de que a catarata e a DMRI possuem um ou mais fato- 
res de risco comuns. Uma segunda possibilidade é a dificuldade de identificar a DMRI na presença de catarata. Uma terceira hipótese é a da exposição à luz, em que com a remoção da catarata, a retina estaria exposta a certos comprimentos de onda ou a maior intensidade de luminosidade, causando dano à retina e aumento do risco de DMRI. Esses autores ainda aceitam a possibilidade da cirurgia de catarata predispor ao desenvolvimento de DMRI, possivelmente através de mecanismo inflamatório, que estaria mais relacionada ao desenvolvimento de DMRI neovascular, com indução de angiogênese pelos macrófagos ${ }^{(7)}$.

\section{Conclusão}

Este trabalho, embora seja retrospectivo, mostra que o mecanismo inflamatório parece não exercer efeito no desenvolvimento de DMRI, uma vez que não foi observado aumento, estatisticamente significante, da incidência desta patologia em olhos que tiveram complicação intra-operatória.

Seria importante que outros estudos fossem conduzidos para esclarecer as dúvidas que ainda existem sobre esta importante patologia.

\section{Abstract}

Puporse: To estimate if a complicated phacoemulsification is a risk factor for developing age related macular degeneration. Methods: Retrospective case-control study with review of records of 1150 patients, that underwent phacoemulsification surgery between 1995 and 1999, performed by a single surgeon (T.C.) Qui square test, Fisher exact test, and Mann-Whitney test were used to perform statistical analysis. Results: There were 224 participants who had cataract surgery on 297 eyes, and from this total, 240 were with no complication and 57 were complicated surgery. In the female group there were a total of 38 complicated surgery, and ten of those developed ARMD. $(p=0,0509)$. In the male group one in 19 of the eyes that have complicated surgery developed ARMD ( $p=0,5737)$. Conclusion: There were no statistically significant increase in the incidence of $A R M D$ in eyes that have complicated surgery, however more research is necessary to clarify the pathogeny of this desease.

Keywords: Macular degeneration/surgery; Cataract extraction; Phacoemulsification/methods; Choroidal neovascularization; Intraoperative complications; Age factors

\section{REFERÊNCIAS}

1. American Academy of Ophthalmology Retina Panel: AgeRelated Macular Degeneration. San Francisco (CA): American Academy of Ophthalmology. 2003. p. 1-30.

2. Klein R, Klein BE, Jensen SC, Cruickshanks KJ. The relationship of ocular factors to the incidence and progression of age-related maculopathy. Arch Ophthalmol. 1998; 116(4):506-13.Comment in: Arch Ophthalmol. 1998; 116(10):1404-5.

3. Klein R, Peto T, Bird A, Vannewkirk MR. The epidemiology of age-related macular degeneration. Am J Ophthalmol. 2004; 137(3): 486-95. Review.

4. Wong TY. Cataract surgery in patients with cataract and age related macular degeneration: do the benefits outweight the risks? Br J Ophthalmol. 2000; 84(12): 1337-8.

5. Lundstrom M, Brege KG, Floren I, Lundh B, Stenevi U, Thorburn W. Cataract surgery and quality of life in patients with age related macular degeneration. Br J Ophthalmol. 2002; 86(12):1330-5.

6. Freeman EE, Munoz B, West SK, Tielsch JM, Schein OD. Is there an association between cataract surgery and age-related macular degeneration? Data from three population-based studies. Am J Ophthalmol. 2003; 135(6):849-56. Comment in: Am J Ophthalmol. 2003; 136(5):961.

7. Mares-Perlman JA, Brady WE, Klein R, Klein BE, Bowen P, Stacewicz-Sapuntzakis M, Palta M. Serum antioxidants and age-related macular degeneration in a population-based casecontrol study. Arch Ophthalmol. 1995; 113(12): 1518-23.

8. Friedman E, Krupsky S, Lane AM, Oak SS, Friedman ES, Egan K, Gragoudas ES. Ocular blood flow velocity in agerelated macular degeneration. Ophthalmology. 1995; 102(4):640-6. Comment in: Ophthalmology. 1995; 102(8):1125-6.

9. Hardy P, Abran D, Li DY, Fernandez H, Varma DR, Chemtob $S$. Free radicals in retinal and choroidal blood flow autoregulation in the piglet: interaction with prostaglandins. Invest Ophthalmol Vis Sci. 1994; 35(2):580-91.

10. Wang JJ, Klein R, Smith W, Klein BE, Tomany S, Mitchell P. Cataract surgery and the 5-year incidence of late-stage agerelated maculopathy: pooled findings from the Beaver Dam and Blue Mountains eye studies. Ophthalmology. 2003; 110(10):1960-7.

11. Lacava AC, De Lucca ES, Centurion V. A cirurgia de catarata em portadores de degeneração macular relacionada à idade (DMRI). Rev Bras Oftalmol. 2000; 59(11): 785-9. 